\title{
Differential Sensitivity of the Species of Candida parapsilosis Sensu Lato Complex Against Statins
}

\author{
Judit Szenzenstein · Attila Gácser · Zsuzsanna Grózer • \\ Zoltán Farkas · Katalin Nagy • Csaba Vágvölgyi • \\ János Márki-Zay • Ilona Pfeiffer
}

Received: 19 February 2013/Accepted: 5 August 2013

(C) Springer Science+Business Media Dordrecht 2013

\begin{abstract}
Candida parapsilosis sensu stricto, Candida orthopsilosis and Candida metapsilosis are human fungal pathogens with clinical importance. The recently reclassified three closely related species have significant variation in virulence, clinical prevalence and susceptibility characteristics to different antifungal compounds. The aim of this study was to investigate the in vitro activity of atorvastatin and fluvastatin against C. metapsilosis, C. orthopsilosis and C. parapsilosis. Susceptibility tests showed that $C$. parapsilosis was the most sensitive while $C$. orthopsilosis was the least susceptible species to both drugs. On the basis of the differential sensitivity, we developed a simple, reliable and highly cost-effective plate assay to distinguish these closely related species. Applying this method, 54 isolates belonging to the $C$. parapsilosis sensu lato complex deposited in Szeged Microbial Collection could be sorted into the three species with $100 \%$ probability.
\end{abstract}

J. Szenzenstein · A. Gácser $(\bowtie) \cdot$ Z. Grózer ·

Z. Farkas · C. Vágvölgyi · I. Pfeiffer

Department of Microbiology, Faculty of Science and

Informatics, University of Szeged, Közép fasor 52,

6726 Szeged, Hungary

e-mail: gacsera@gmail.com

K. Nagy

Faculty of Dentistry, University of Szeged,

Tisza L. krt. 64, 6720 Szeged, Hungary

J. Márki-Zay

Solvo Biotechnology, Szeged, Hungary
Keywords Atorvastatin - Fluvastatin . Candida metapsilosis $\cdot$ C. orthopsilosis .

C. parapsilosis

\section{Introduction}

Molecular studies of the Candida parapsilosis subtypes recently led to their separation into different, closely related species: the more prevalent C. parapsilosis sensu stricto, Candida orthopsilosis and Candida metapsilosis [1]. Human diseases caused by C. parapsilosis complex have significantly increased in importance and prevalence over the last decade $[2,3]$. Recently, survey data from different hospitals $[4,5]$ show that $C$. parapsilosis sensu stricto is the second or third most common Candida spp. isolated from blood cultures. The examination of the distribution of the strains within the $C$. parapsilosis complex from different isolates obtained from blood and other sterile sources in European tertiary-care hospital revealed that the majority of the isolates (111 of 122) were $C$. parapsilosis sensu stricto, whereas significantly fewer (10 of 122) were identified as C. orthopsilosis and only one isolate was identified as C. metapsilosis [4]. This has been observed in other studies [5].

The sensitivity of the three species to antifungal drugs is different $[4,5]$, indicating that accurate identification would influence therapy. Conventional laboratory methods are unable to differentiate the 
three species, and although molecular methods are rapid and sensitive, they are not routinely available.

Statins were originally identified as fungal metabolites [6] that act as selective inhibitors of the 3-hydroxy-3-methylglutaryl-coenzyme A reductase [7], which is the enzyme responsible for the conversion of hydroxy-methylglutaryl-coenzyme A into mevalonic acid. In addition to their cholesterollowering effects, statins possess antifungal activity through inhibiting the formation of ergosterol, which is a key component of the cell membrane [8-10]. The minimal inhibitory concentrations of current statins against pathogenic fungi and yeasts are rather high; hence, they are not clinically useful as the required dose for a therapeutic effect would be associated with unacceptable serious, toxic side effects. However, statins can potentially serve to differentiate Candida species based on susceptibility profiles.

In the present study, the susceptibility of 13 strains of C. metapsilosis, 20 of C. orthopsilosis and 21 of C. parapsilosis sensu stricto to atorvastatin and fluvastatin was examined.

\section{Materials and Methods}

\section{Yeast Strains and Cultivation}

Strains used in these experiments are listed in Table 1. They were maintained on YPD (1\% glucose, $1 \%$ peptone, $0.5 \%$ yeast extract, $2 \%$ agar) medium at $4{ }^{\circ} \mathrm{C}$. Clinical isolates originated from Hungarian hospitals were identified by ITS sequencing in a previous work [11].

\section{Statins}

Atorvastatin (Atoris, KRKA) and fluvastatin (Lescol, Novartis) were of pharmaceutical grade. Stock solutions were prepared by dissolving the drugs in methanol and then stored at $-80{ }^{\circ} \mathrm{C}$. The concentration of the stock solutions was as follows: atorvastatin $20 \mathrm{mg} \mathrm{mL}^{-1}$, and fluvastatin $40 \mathrm{mg} \mathrm{mL}^{-1}$.

\section{In Vitro Susceptibility Test}

The in vitro antimycotic effect of atorvastatin and fluvastatin was determined by a microdilution method.
The stock solution of statins was diluted in yeast nitrogen base (YNB) without amino acids (DIFCO) liquid medium and applied in final concentrations of $50-1.56 \mu \mathrm{g} \mathrm{mL}^{-1}$ in the case of atorvastatin and $25-0.78 \mu \mathrm{g} \mathrm{mL}^{-1}$ in the case of fluvastatin. The experiments were carried out in final volume of $100 \mu \mathrm{L}$ in $96-w e l l$ microplates. Yeast nitrogen base medium was prepared as suggested by the supplier and contained $1 \%$ glucose as the carbon source. Yeast cell inoculates were prepared from 2-day-old cultures cultivated in YPD liquid medium at $30{ }^{\circ} \mathrm{C}$ with vigorous shaking. The cells were washed with sterile distilled water, and after repeated washing, they were suspended in YNB. The initial cell concentration in each well was adjusted to $4 \times 10^{4}$ cells $\mathrm{mL}^{-1}$.

The microplate cultures were then grown at $30^{\circ} \mathrm{C}$ for $48 \mathrm{~h}$ with shaking in a BIOTEK SynergyHT shaker. Growth was detected by checking the optical density (OD) of the cultures at $600 \mathrm{~nm}$ in every $5 \mathrm{~min}$ (Gen5 software). Non-inoculated medium was used as background, and the growth control contained inoculated YNB medium (initial cell concentration was $4 \times 10^{4}$ cells $\mathrm{mL}^{-1}$ ). A growth control containing the same amount of methanol as the statin-containing samples was also applied.

The experiments were repeated two times, each plate carried two parallels per each strain and each statin.

\section{Plate Assay}

Strains cultivated for $48 \mathrm{~h}$ at $30{ }^{\circ} \mathrm{C}$ in YPD medium were diluted in YNB medium, and $10^{5}, 10^{4}, 10^{3}$ and $10^{2}$ cells were spotted onto YNB plates containing 30 , 40 and $50 \mu \mathrm{g} \mathrm{mL}^{-1}$ fluvastatin or atorvastatin, respectively. The plates were incubated at $30{ }^{\circ} \mathrm{C}$ for 5 days.

\section{Results and Discussion}

The in vitro minimal inhibitory concentration (MIC) of atorvastatin and fluvastatin against the species of C. parapsilosis sensu lato complex was studied by microdilution method. Three strains of each species were included in the study: C. metapsilosis SZMC 8022, SZMC 1547, SZMC 1548; C. orthopsilosis SZMC 1545, SZMC 8119, SZMC 8121; and $C$. parapsilosis CBS 6318, SZMC 1577, SZMC 8002. 
Table 1 Candida isolates

\begin{tabular}{|c|c|c|c|}
\hline & Species & Strain no. & Isolation place \\
\hline 1 & C. parapsilosis & SZMC 1361 & Blood, Debrecen, Hungary \\
\hline 2 & C. parapsilosis & SZMC 1438 & Blood, Debrecen, Hungary \\
\hline 3 & C. parapsilosis & SZMC 8043C & Stomach, Pécs, Hungary \\
\hline 4 & C. parapsilosis & SZMC 8045 & Blood, Pécs, Hungary \\
\hline 5 & C. parapsilosis & SZMC 1577 & Szeged, Hungary \\
\hline 6 & C. parapsilosis & SZMC 1572 & Szeged, Hungary \\
\hline 7 & C. parapsilosis & SZMC 1568 & Szeged, Hungary \\
\hline 8 & C. parapsilosis & SZMC 8050C & Szeged, Hungary \\
\hline 9 & C. parapsilosis & SZMC 1587 & Szeged, Hungary \\
\hline 10 & C. parapsilosis & SZMC 8051C & Szeged, Hungary \\
\hline 11 & C. parapsilosis & SZMC 1569 & Szeged, Hungary \\
\hline 12 & C. parapsilosis & SZMC 8004 & Ear, Debrecen, Hungary \\
\hline 13 & C. parapsilosis & SZMC 1594 & Cannula, Debrecen, Hungary \\
\hline 14 & C. parapsilosis & SZMC 1590 & Urine, Debrecen, Hungary \\
\hline 15 & C. parapsilosis & SZMC 1592 & Sputum, Debrecen, Hungary \\
\hline 16 & C. parapsilosis & SZMC 8002 & Ear, Debrecen, Hungary \\
\hline 17 & C. parapsilosis & SZMC 1596 & Wound, Debrecen, Hungary \\
\hline 18 & C. parapsilosis & CBS 1954 & Olive, Italy \\
\hline 19 & C. parapsilosis & CBS 6318 & Olive \\
\hline 20 & C. parapsilosis & SZMC 8112 & Germany \\
\hline 21 & C. parapsilosis & SZMC 8113 & Sputum, Cagliari, Italy \\
\hline 22 & C. metapsilosis & SZMC 8029 & Blood, Debrecen, Hungary \\
\hline 23 & C. metapsilosis & SZMC 1547 & Unknown \\
\hline 24 & C. metapsilosis & SZMC 1548 & Unknown \\
\hline 25 & C. metapsilosis & SZMC 8022 & Throat, Pécs, Hungary \\
\hline 26 & C. metapsilosis & SZMC 8091 & Sputum, Pisa, Italy \\
\hline 27 & C. metapsilosis & SZMC 8092 & Bronchial aspirate, Pisa, Italy \\
\hline 28 & C. metapsilosis & SZMC 8093 & Nail, Pisa, Italy \\
\hline 29 & C. metapsilosis & SZMC 8094 & Feces, Pisa, Italy \\
\hline 30 & C. metapsilosis & SZMC 8095 & Nail, Pisa, Italy \\
\hline 31 & C. metapsilosis & SZMC 8096 & Nail, Pisa, Italy \\
\hline 32 & C. metapsilosis & SZMC 8097 & Feces, Pisa, Italy \\
\hline 33 & C. metapsilosis & SZMC 8098 & Feces, Pisa, Italy \\
\hline 34 & C. metapsilosis & SZMC 8099 & Peripheral blood, Auckland, New Zealand \\
\hline 35 & C. orthopsilosis & SZMC 1545 & Unknown \\
\hline 36 & C. orthopsilosis & SZMC 1546 & Unknown \\
\hline 37 & C. orthopsilosis & SZMC 8115 & Germany \\
\hline 38 & C. orthopsilosis & SZMC 8116 & Germany \\
\hline 39 & C. orthopsilosis & SZMC 8117 & Germany \\
\hline 40 & C. orthopsilosis & SZMC 8118 & Germany \\
\hline 41 & C. orthopsilosis & SZMC 8119 & Germany \\
\hline 42 & C. orthopsilosis & SZMC 8120 & Germany \\
\hline 43 & C. orthopsilosis & SZMC 8121 & Germany \\
\hline 44 & C. orthopsilosis & SZMC 8122 & Germany \\
\hline 45 & C. orthopsilosis & SZMC 8100 & Pisa, Italy, nail \\
\hline
\end{tabular}


Table 1 continued

\begin{tabular}{llll}
\hline & Species & Strain no. & Isolation place \\
\hline 46 & C. orthopsilosis & SZMC 8101 & Pisa, Italy, skin \\
47 & C. orthopsilosis & SZMC 8102 & L' Aquila, Italy, catheter \\
48 & C. orthopsilosis & SZMC 8103 & Pisa, Italy, bronchial aspirate \\
49 & C. orthopsilosis & SZMC 8104 & Pisa, Italy, nail \\
50 & C. orthopsilosis & SZMC 8105 & Pisa, Italy, bronchial aspirate \\
51 & C. orthopsilosis & SZMC 8106 & NCPF, UK, unknown \\
52 & C. orthopsilosis & SZMC 8107 & Pisa, Italy, skin \\
53 & C. orthopsilosis & SZMC 8108 & Pisa, Italy, sputum \\
54 & C. orthopsilosis & SZMC 8109 & Pisa, Italy, catheter \\
\hline
\end{tabular}

The applied concentration range was between 50 and $1.56 \mu \mathrm{g} \mathrm{mL}^{-1}$ for atorvastatin (Fig. 1) and $25-0.78 \mu \mathrm{g} \mathrm{mL}^{-1}$ for fluvastatin (Fig. 2). The antifungal activity of the atorvastatin varied; $C$. parapsilosis was the most sensitive species while the susceptibility of C. orthopsilosis and C. metapsilosis was similar (Fig. 1). The $\mathrm{MIC}_{100}$ value (100\% growth inhibition) of atorvastatin was $25 \mu \mathrm{g} \mathrm{mL}^{-1}$ for C. parapsilosis
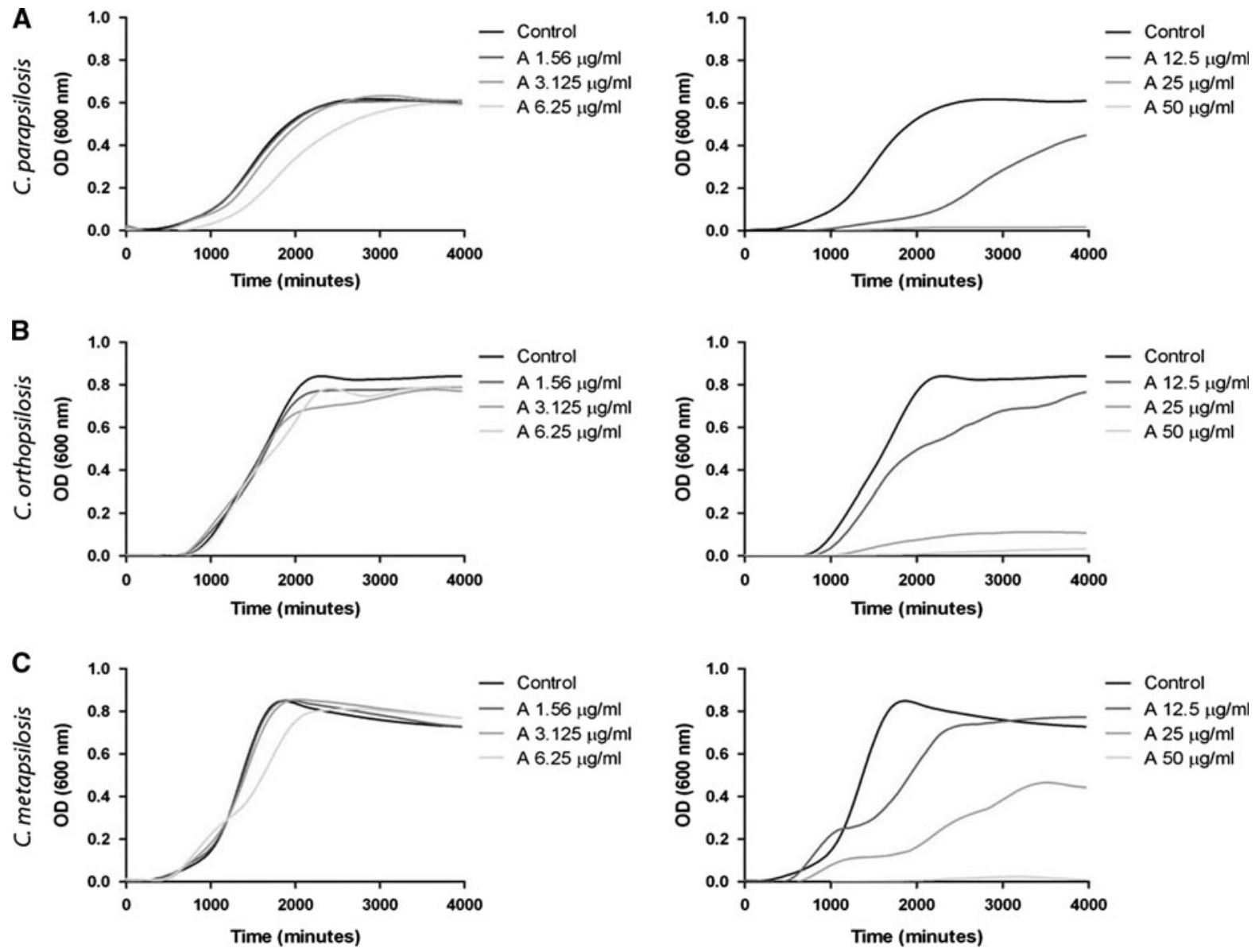

Fig. 1 Growth curves of three representative isolates of C. parapsilosis (a), C. orthopsilosis (b) and C. metapsilosis (c) in the presence of different concentrations of atorvastatin, measured in 5-min intervals for $66 \mathrm{~h}$ 

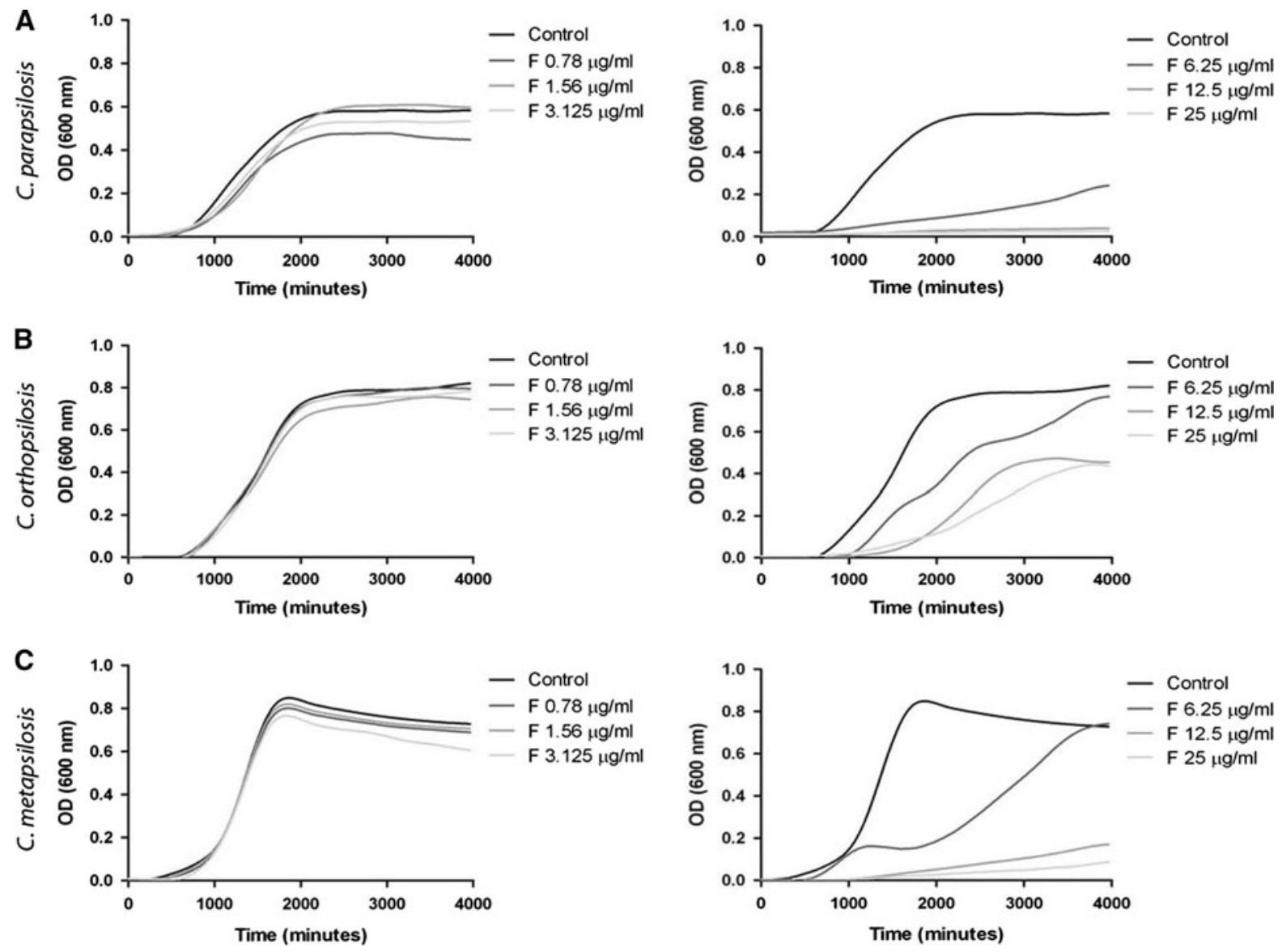

Fig. 2 Growth curves of three representative isolates of C. parapsilosis (a), C. orthopsilosis (b) and C. metapsilosis (c) in the presence of different concentrations of fluvastatin, measured in 5-min intervals for $66 \mathrm{~h}$

(Fig. 1a) and $50 \mu \mathrm{g} \mathrm{mL}^{-1}$ for C. orthopsilosis and $C$. metapsilosis (Fig. 1b, c).

Remarkable differences were detected in the sensitivity to fluvastatin: $C$. parapsilosis was the most susceptible species, while $C$. orthopsilosis proved the less sensitive to it (Fig. 2). Fluvastatin was more effective than atorvastatin, as it completely inhibited the growth of $C$. parapsilosis at $12.5 \mu \mathrm{g} \mathrm{mL}^{-1}$ concentration (Fig. 2a). C. orthopsilosis was the least susceptible to fluvastatin (Fig. 2b) as $12.5 \mu \mathrm{g} \mathrm{mL}^{-1}$ concentration caused only $50 \%$ growth inhibition. In the case of $C$. metapsilosis, $90 \%$ inhibition was demonstrated at this concentration (Fig. 2c).

On the basis of the data presented above, we tested the sensitivity of our strains of the three species on solidified YNB medium supplemented with 30, 40 and $50 \mu \mathrm{g} \mathrm{mL}^{-1}$ fluvastatin or atorvastatin. After 5 days of incubation at $30^{\circ} \mathrm{C}$, none of the 21 strains of
C. parapsilosis sensu stricto could grow on medium containing the lowest concentration, $30 \mu \mathrm{g} \mathrm{mL}^{-1}$, of either fluvastatin or atorvastatin. In contrast, the 20 strains of $C$. orthopsilosis and the 13 strains of $C$. metapsilosis formed colonies at $50 \mu \mathrm{g} \mathrm{mL}^{-1}$ of atorvastatin. At $30 \mu \mathrm{g} \mathrm{mL}^{-1}$ of fluvastatin, C. orthopsilosis cells grew with inoculates as low as $10^{2}$ cells (Fig. 3b), whereas C. metapsilosis colonies were detected only at the highest cell densities (Fig. 3c). Hence, fluvastatin at $30 \mu \mathrm{g} \mathrm{mL}^{-1}$ concentration was sufficient to discriminate between $C$. metapsilosis and C. orthopsilosis using different inoculums of these species. Based on these results, fluvastatin was significantly more discriminatory than atorvastatin.

Species differentiation is laborious in the case of closely related human pathogenic fungi or yeasts. Several conventional and molecular methods exist, but all of them have their limits, so new reliable 
A

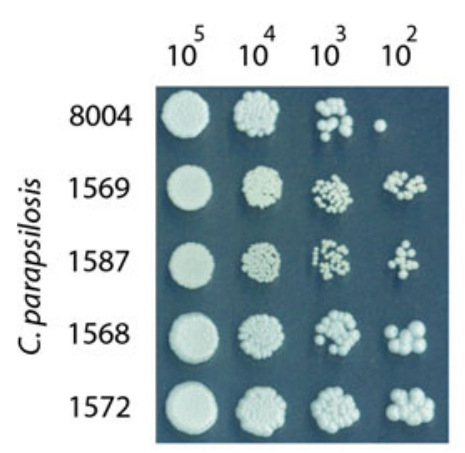

B

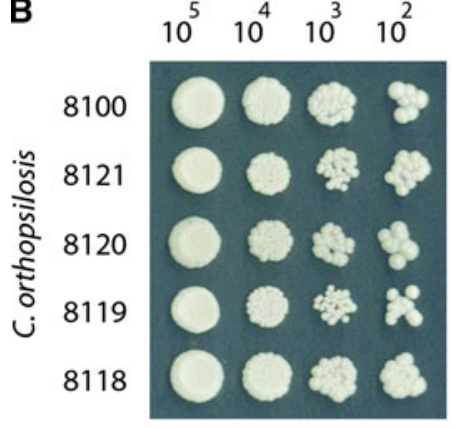

C

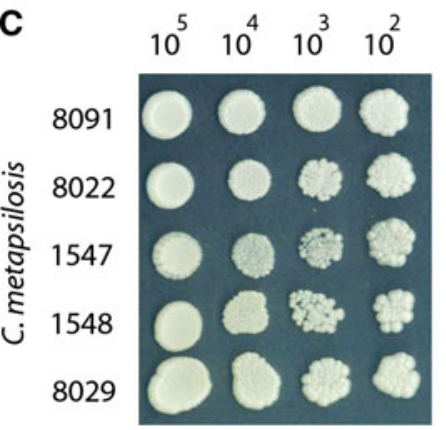

Fluvastatin $30 \mu \mathrm{g} / \mathrm{ml}$
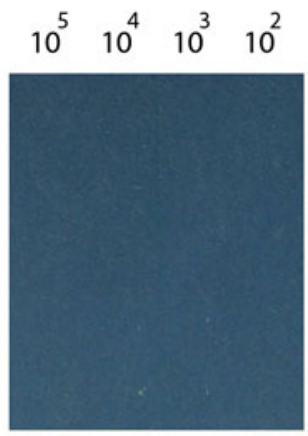

$10^{5} \quad 10^{4} \quad 10^{3} \quad 10^{2}$

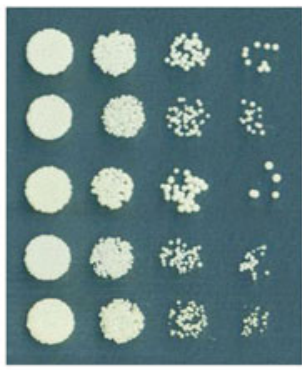

$10^{5} \quad 10^{4} \quad 10^{3} \quad 10^{2}$

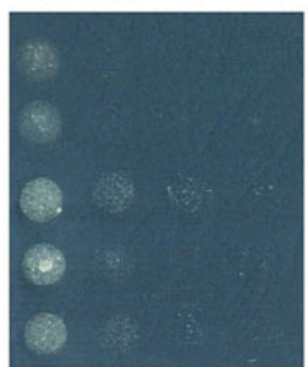

Control

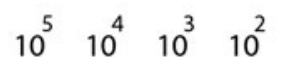

8113

1577

8045

1438

1361

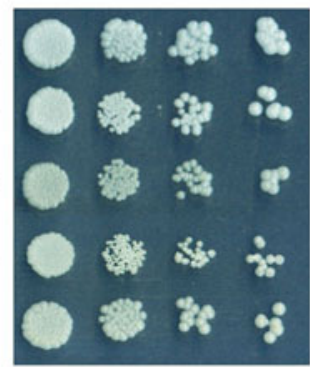

$10^{5} \quad 10^{4} \quad 10^{3} \quad 10^{2}$

8117

8116

8115

1546

1545

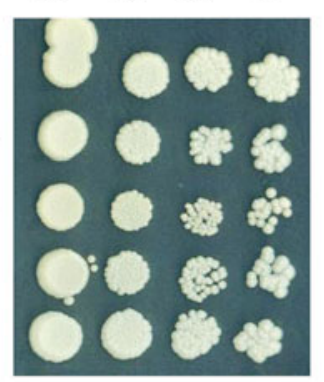

$10^{5} \quad 10^{4} \quad 10^{3} \quad 10^{2}$

8092

8093

8094

8095

8096

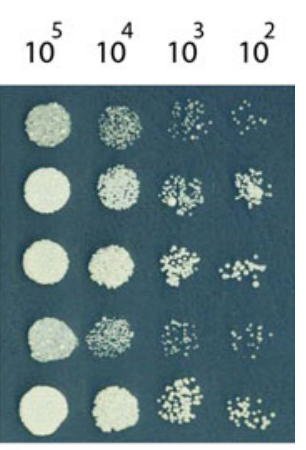

Fluvastatin $30 \mu \mathrm{g} / \mathrm{ml}$

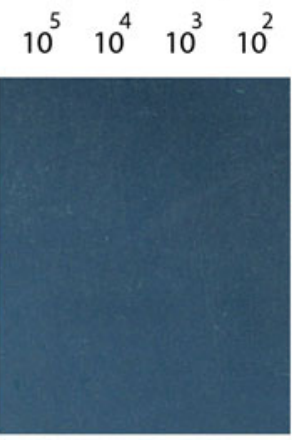

Fig. 3 Colony formation of ten representative strains of C. parapsilosis (a), C. orthopsilosis (b) and C. metapsilosis (c) on solid YNB medium (control) and YNB supplemented with $30 \mu \mathrm{g} \mathrm{m}^{-1}$ fluvastatin

approaches are required. Differences in lovastatin sensitivity have been used for species selection in Rhizomucor genus in a recent study [12]. Based on the remarkable difference observed in the susceptibilities of $C$. parapsilosis, C. orthopsilosis and C. metapsilosis to fluvastatin, we propose a simple plate assay to distinguish the strains of the three species. Our data indicate that a dilution series of the isolated strain should be inoculated on YNB medium containing $30 \mu \mathrm{g} \mathrm{mL}^{-1}$ fluvastatin. C. parapsilosis strains should not grow under this condition (Fig. 3a). As C. orthopsilosis is the most resistant species to this drug, robust growth should occur at each dilution (Fig. 3b), whereas only the dilutions with $10^{4}$ or $10^{5}$ of $C$. metapsilosis will have growth (Fig. 3c). The method was validated with 13 strains of C. metapsilosis, 20 C. orthopsilosis and 21 C. parapsilosis sensu stricto. In conclusion, this method provides a simple and effective method for differentiating these three closely related species, and this approach can be used as an alternative technique for definite identification of $C$. parapsilosis sensu lato isolates, which will be a useful tool for future epidemiological studies. 
Acknowledgments This work was supported by the Hungarian National Office for Research and Technology grant: TECH08-A1IVDMDQ08. AG was supported in part by OTKA NF 84006 and by EMBO Installation Grant 1813. JSZ was supported by TÁMOP 4.2.4.A/2-11-1-2012-0001. This research was realized in the frames of TÁMOP 4.2.4. A/2-11-1-2012-0001 "National Excellence Program-Elaborating and operating an inland student and researcher personal support system convergence program." The project was subsidized by the European Union and cofinanced by the European Social Fund. Authors are grateful to Dr. Oliver Bader, Dr. Ariana Tavanti and Dr. László Majoros for providing the clinical isolates of Candida species.

\section{References}

1. Tavanti A, Davidson AD, Gow NA, Maiden MC, Odds FC. Candida orthopsilosis and Candida metapsilosis spp. nov. to replace Candida parapsilosis groups II and III. J Clin Microbiol. 2005;43(1):284-92. doi:10.1128/JCM.43.1.284292.2005 .

2. Trofa D, Gacser A, Nosanchuk JD. Candida parapsilosis, an emerging fungal pathogen. Clin Microbiol Rev. 2008;21(4): 606-25. doi:10.1128/CMR.00013-08.

3. van Asbeck EC, Clemons KV, Stevens DA. Candida parapsilosis: a review of its epidemiology, pathogenesis, clinical aspects, typing and antimicrobial susceptibility. Crit Rev Microbiol. 2009;35(4):283-309. doi:10.3109/104084 10903213393.

4. de Toro M, Torres MJ, Maite R, Aznar J. Characterization of Candida parapsilosis complex isolates. Clin Microbiol Infect. 2010. doi:10.1111/j.1469-0691.2010.03302.x.
5. Thierry G, Morio F, Le Pape P, Gay-Andrieu F, Barre O, Miegeville M. Prevalence of Candida parapsilosis, C. orthopsilosis and C. metapsilosis in candidemia over a 5-year period at Nantes hospital and in vitro susceptibility to three echinocandins by E-test ${ }^{\circledR}$. Pathol Biol (Paris). 2011;59(1): 52-6. doi:10.1016/j.patbio.2010.07.019.

6. Endo A. A gift from nature: the birth of the statins. Nat Med. 2008;14(10):1050-2. doi:10.1038/nm1008-1050.

7. Stancu C, Sima A. Statins: mechanism of action and effects. J Cell Mol Med. 2001;5(4):378-87.

8. Macreadie IG, Johnson G, Schlosser T, Macreadie PI. Growth inhibition of Candida species and Aspergillus fumigatus by statins. FEMS Microbiol Lett. 2006;262(1): 9-13. doi:10.1111/j.1574-6968.2006.00370.x.

9. Nash JD, Burgess DS, Talbert RL. Effect of fluvastatin and pravastatin, HMG-CoA reductase inhibitors, on fluconazole activity against Candida albicans. J Med Microbiol. 2002;51(2):105-9.

10. Song JL, Lyons CN, Holleman S, Oliver BG, White TC. Antifungal activity of fluconazole in combination with lovastatin and their effects on gene expression in the ergosterol and prenylation pathways in Candida albicans. Med Mycol. 2003;41(5):417-25.

11. Kocsube S, Toth M, Vagvolgyi C, Doczi I, Pesti M, Pocsi I, et al. Occurrence and genetic variability of Candida parapsilosis sensu lato in Hungary. J Med Microbiol. 2007; 56(Pt 2):190-5. doi:10.1099/jmm.0.46838-0.

12. Liao JK, Laufs U. Pleiotropic effects of statins. Annu Rev Pharmacol Toxicol. 2005;45:89-118. doi:10.1146/annurev. pharmtox.45.120403.095748. 